\title{
Focusing inside Disordered Media with the Generalized Wigner-Smith Operator
}

\author{
Philipp Ambichl, ${ }^{1}$ Andre Brandstötter, ${ }^{1}$ Julian Böhm, ${ }^{2}$ Matthias Kühmayer, ${ }^{1}$ Ulrich Kuhl, ${ }^{2}$ and Stefan Rotter ${ }^{1}$ \\ ${ }^{1}$ Institute for Theoretical Physics, Vienna University of Technology (TU Wien), A-1040 Vienna, Austria, EU \\ ${ }^{2}$ Université Côte d'Azur, CNRS, LPMC, 06108 Nice, France, EU
}

(Received 13 March 2017; published 18 July 2017)

\begin{abstract}
We introduce a wave front shaping protocol for focusing inside disordered media based on a generalization of the established Wigner-Smith time-delay operator. The key ingredient for our approach is the scattering (or transmission) matrix of the medium and its derivative with respect to the position of the target one aims to focus on. A specific experimental realization in the microwave regime is presented showing that the eigenstates of a corresponding operator are sorted by their focusing strength—ranging from strongly focusing on the designated target to completely bypassing it. Our protocol works without optimization or phase conjugation and we expect it to be particularly attractive for optical imaging in disordered media.
\end{abstract}

DOI: 10.1103/PhysRevLett.119.033903

One of the most formidable challenges for imaging in complex environments is to overcome the limitations imposed by the presence of disorder. In particular, if the wave scattering induced by a disordered medium is strong enough to suppress the ballistic contribution in the imaging process entirely, seeing through this medium or focusing on a target inside of it becomes a highly nontrivial exercise- $a$ difficulty that is particularly evident in the field of biological and medical imaging. A promising new approach to image and focus also in the regime of multiple scattering is to exploit the information stored in a system's scattering matrix $[1,2]$. In this emerging new field of "wave front shaping" $[3,4]$ spectacular advances have recently been made, such as to focus light behind an opaque layer [5-9], or to send and retrieve images across it [10-14]. Also, thanks to these advances the focusing of light inside highly disordered media could recently be demonstrated using embedded fluorescent probes and nanocrystals [15-17] or using digital optical phase conjugation to focus light onto a target moving inside an otherwise static environment [18-21].

Here we present a new approach for focusing inside a disordered material that has the considerable advantage of working without the requirement to implant a fluorescent body at the focus or to phase conjugate a wave scattered at the focus position. Our technique also allows us to tune the degree of focus on a designated position inside the disorder, including the case where the target is entirely avoided by the scattered wave front. Our starting point for achieving this goal is the time-delay operator $Q$ introduced by Eugene Wigner and Felix Smith [22,23]. Originally devised for

Published by the American Physical Society under the terms of the Creative Commons Attribution 4.0 International license. Further distribution of this work must maintain attribution to the author(s) and the published article's title, journal citation, and DOI. nuclear scattering problems to deduce the time associated with a scattering event from stationary measurements of the asymptotic scattering amplitudes, this concept prominently resurfaced in mesoscopic physics [24] and very recently in attosecond physics [25] as well as in the newly emerging community of wave front shaping [4,26-30].

The Wigner-Smith time-delay operator $Q$ is constructed based on a system's scattering matrix $S$ by way of a frequency derivative, $Q=-i S^{-1} d S / d \omega$. The eigenvalues of $Q$, also called "proper delay times," measure the time delay associated with the scattering by a given potential [4,31-33]. The corresponding eigenvectors, also called "principal modes," are states that can be associated with this well-defined time-delay-a property that makes them dispersion-free [26] in the sense that a small variation of their input frequency does not change their spatial output profile. Moreover, principal modes have been shown to be "particlelike" in situations where ballistic scattering occurs [27]. The potential for applications of these states as efficient communication channels in systems with multiple in- and output ports has recently taken center stage when first experiments reported on the successful implementation of principal modes in optical multimode fibers $[28,29]$ as well as in resonant scattering media [30,34].

Here we demonstrate that the concept underlying the principal modes is not at all restricted to the time-delay operator $Q$ from above; specifically, we show that in the same way as the conventional principal modes are invariant with respect to a frequency variation, we may also create wave states that are invariant with respect to changes in the system configuration, like a local shift of a designated scatterer inside a disordered medium. While the frequencyinsensitive principal modes are the eigenstates of the timedelay operator $Q=-i S^{-1} d S / d \omega$ (involving a frequency derivative), the class of states we introduce will be the eigenstates of a corresponding operator $Q_{\alpha}=-i S^{-1} d S / d \alpha$, where the parameter $\alpha$ stands, e.g., for the position of a 
movable scatterer. What is special about our new approach is that the eigenstates of $Q_{\alpha}$ are not only insensitive to a small change of $\alpha$, but that the associated eigenvalues indicate how strongly the corresponding conjugate quantity to $\alpha$ is affected by the scattering process. This insight allows us to maximally focus on or maximally avoid a specific target inside multiple scattering media just based on the medium's scattering matrix $S$ and its variation due to a shift of the target particle that one aims to focus on.

We start by reviewing the principal modes' remarkable property of being insensitive with respect to a change in frequency of the incident wave. The corresponding eigenvalue equation for the principal modes $\vec{u}_{n}$ (given as a coefficient vector in a certain basis) and for the proper delay times $\theta_{n}$ reads as follows:

$$
Q \vec{u}_{n}=-i S^{-1} \frac{d S}{d \omega} \vec{u}_{n}=\theta_{n} \vec{u}_{n},
$$

with $\omega$ being the frequency. In a waveguide system, as shown in Fig. 1, the incident principal modes can be decomposed into waves injected from the left and right lead, respectively, i.e., $\vec{u}_{n}=\left(\vec{u}_{n, L}, \vec{u}_{n, R}\right)^{T}$. For a unitary scattering matrix, $S^{\dagger} S=\mathbb{1}$, the time-delay operator $Q$ is Hermitian with real eigenvalues $\theta_{n}$. Using the input-output relation $\vec{v}_{n}(\omega)=S(\omega) \vec{u}_{n}$, we can rewrite Eq. (1) for a (static) time-delay eigenstate $\vec{u}_{n}$ evaluated at a chosen frequency $\omega_{0}$

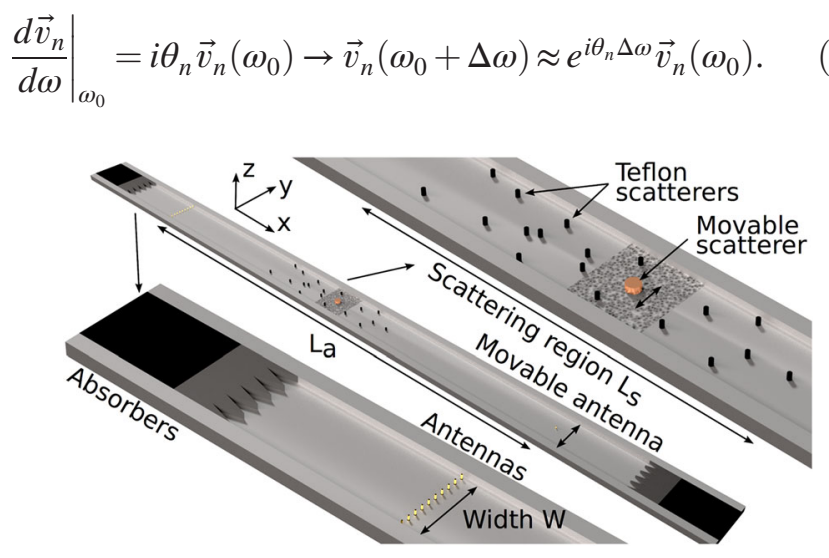

FIG. 1. Sketch of the experimental setup. The system consists of a rectangular aluminum waveguide of height $H=8 \mathrm{~mm}$, width $W=10 \mathrm{~cm}$, and total length $L=2.38 \mathrm{~m}$, see middle panel. The top plate (not shown) can be removed. The wave front is injected from the left using ten monopole antennas, see bottom panel. The scattering region displayed in the top panel has a length $L_{s}=$ $60 \mathrm{~cm}$ and consists of 18 cylindrical Teflon scatterers (black cylinders, index of refraction $n=1.44$, radius $2.55 \mathrm{~mm}$, height $8 \mathrm{~mm}$ ). A (re-)movable brass scatterer of radius $8.825 \mathrm{~mm}$ and height $8 \mathrm{~mm}$ is located in the central part of the scattering region. The placement of scatterers in this sketch was randomly generated and matches the actual scatterer positions in the experiment. The distance between the injecting antenna array and the scanning antenna is $L_{a}=1.50 \mathrm{~m}$. The grained area around the movable scatterer indicates the region shown in Figs. 2 and 3.
The expression on the right-hand side of Eq. (2) states that the output vector of a principal mode, $\vec{v}_{n}=\left(\vec{v}_{n, L}, \vec{v}_{n, R}\right)^{T}$, retains its original orientation when shifting the input frequency in the vicinity of $\omega_{0}$. This, in turn, translates into output patterns that are invariant (to first order) with respect to a frequency change (apart from a complex global factor). An aspect of the above derivation that has so far been unexploited is the fact that the derivative in Eq. (1) does not necessarily have to be taken with respect to the frequency. In other words: the symbol $\omega$ can in principle represent any other parametric dependence of the scattering matrix and the stability property of the corresponding principal modes will still hold with respect to a variation of this new parameter. Accordingly, we shift our attention to a whole class of generalized Wigner-Smith (GWS) operators $Q_{\alpha}=-i S^{-1} d S / d \alpha$ in which the frequency $\omega$ is replaced by the arbitrary parameter $\alpha$. We also expect that these GWS operators may have interesting connections to earlier works where statistical properties of parametric variations of the scattering matrix have been studied [35-37].

While the eigenstates of $Q_{\alpha}$ are invariant with respect to a small parametric shift of $\alpha$ already by construction [as in Eq. (2) with $\omega \rightarrow \alpha$ ], we still need to clarify how to interpret the corresponding eigenvalues $\theta_{n}^{\alpha}$. Already from the dimensions it is clear that $\theta_{n}^{\alpha}$ must be associated with the conjugate variable to $\alpha$, in the same way as the delay time $\theta$ is the conjugate quantity to the frequency $\omega$. To make this more evident we now define $C_{\alpha}:=-i d / d \alpha$ as the corresponding conjugate operator to $\alpha$ and assume that $\alpha$ stands for a global variable. It follows after a short derivation (see Supplemental Material [38]),

$$
\vec{u}^{\dagger} Q_{\alpha} \vec{u}=\vec{u}^{\dagger} C_{\alpha} \vec{u}-\vec{v}^{\dagger} C_{\alpha} \vec{v}=\left\langle C_{\alpha}\right\rangle_{\text {in }}-\left\langle C_{\alpha}\right\rangle_{\text {out }},
$$

where $\vec{v}(\alpha)=S(\alpha) \vec{u}$ is the output vector and $\langle\cdots\rangle_{\text {in/out }}$ denotes the expectation value evaluated in the input or output scattering state. Following Eq. (3), $Q_{\alpha}$ is the appropriate operator to measure the shift in the conjugate variable to $\alpha$, which the wave experiences due to the scattering process-in perfect analogy to the time-delay operator measuring a shift in time, i.e., in the conjugate quantity of the frequency $\omega$. One specific example for such a global parameter $\alpha$ could be the displacement in $y$ direction (i.e., $\alpha=y$ ) of the entire scattering landscape from its initial position. The conjugate variable to the position is the momentum such that the eigenvalue $\theta_{n}^{y}$ of the operator $Q_{y}$ measures the momentum shift (in the $y$ direction) that the corresponding eigenstates experience as a result of scattering at the entire potential landscape. Solving the eigenvalue problem for the operator $Q_{y}$ thus provides us with an orthogonal and complete basis of eigenstates that are sorted by this momentum shift. To be more precise, the operator $Q_{y}$ measures the shift in the 
wave number in the displacement direction, but for the sake of simplicity we refer to this shift as momentum shift $\Delta k$.

In the present context, we will be specifically interested in the case where $\alpha$ is the transverse position $y_{i}$ of the $i$ th scattering element inside a strongly disordered medium consisting of altogether $i_{\text {tot }} \geq i$ such individual elements (see Fig. 1). In this case, $\alpha$ does not represent a global variable since only the $i$ th scatterer is shifted rather than the whole system. It turns out that Eq. (3) still remains valid for this more general case, but that the corresponding expectation values are then evaluated based on the local field amplitudes in the vicinity of the $i$ th scatterer (see Supplemental Material [38]). The eigenvalues $\theta_{n}^{y_{i}}$ of $Q_{y_{i}}$ are thus equal to the momentum shift $\Delta k^{i}$ that the wave experiences locally when colliding with the $i$ th scatterer (in the presence of all other scatterers and boundaries of the medium). Note that a large momentum shift requires that the wave front of the incident eigenstate is backscattered significantly at the target position, which is equivalent to focusing on this scatterer. On the other hand, when the wave does not get scattered at the target at all (e.g., by not reaching the $i$ th scatterer), the momentum transfer will, correspondingly, be very small. In this way the GWS operator $Q_{y_{i}}$ provides us the means to focus on a designated scatterer inside a disordered medium or to omit this target simply by generating an eigenvector of the GWS operator corresponding to a large or small eigenvalue, respectively.

To demonstrate the efficiency of this approach also in the experiment, we implemented a microwave scattering setup as displayed in Fig. 1, consisting of a rectangular multimode waveguide made of aluminum with dimensions $L \times W \times H=2.38 \mathrm{~m} \times 10 \mathrm{~cm} \times 8 \mathrm{~mm}$ (see caption in Fig. 1). Additional absorbers (types: LS-14 and LS-16 from EMERSON\&CUMING) at the ends of the waveguide mimic semi-infinite leads. Ten antennas are placed equidistantly in the incident part of the waveguide controlling ten transmission channels individually. The antennas are addressed by IQ modulators (GTM 1 M2L-68A-5 of GT Microwave Inc.), which allow us to adjust the phase and amplitude of the signal emitted by each antenna. At the operation frequency of $15.5 \mathrm{GHz}$, ten transverse electric modes are excited (shaping the incident wave front is explained in more detail in Ref. [42]). The array of emitting antennas is connected via a power splitter (Microot MPD16-060180) to a 4 port vector network analyzer (VNA, Agilent E5071C). The transmission through the scattering system is measured with a single movable antenna placed at the output side of the waveguide. In the middle we place 18 cylindrical Teflon scatterers of radius $r=2.55 \mathrm{~mm}$ forming the disordered background and one brass scatterer of radius $r_{b}=8.825 \mathrm{~mm}$ being the designated scatterer that we shift to evaluate the spatial derivative in the GWS operator. The relatively high refractive index of the Teflon elements $(n=1.44)$ lets all waves undergo multiple scattering events before being transmitted. Both the material and the increased size of the targeted brass scatterer are chosen to increase the signal-tonoise ratio of the signal its shift leaves in the measured scattering amplitudes. In Ref. [38] we also provide numerical simulations to demonstrate the applicability of our concept for the case when all scatterers are made of Teflon (or brass) and many more than ten modes are involved. Note that we stay away from the regime of Anderson localization where wave control is very limited [43]. In the following, the parameter $\alpha$ corresponds to the transverse position $y_{b}$ of the central brass scatterer and the operator $Q_{y_{b}}$ can be computed from the shift $\pm \Delta y_{b}$ from its initial position, which we choose in the experiment to be of the same size as the in-plane radius of the brass scatterer.

As in most experiments [1,2], we also only have access to a subpart of the entire scattering matrix. Specifically, we can measure only the $10 \times 10$ transmission matrix $t$, where the complex matrix elements $t_{j i}$ stand for the transmission of the $i$ th antenna of the input antenna array to the $j$ th position of the movable antenna at the output. Even for flux-conserving scattering (without gain or loss), the $t$ matrix is generally nonunitary, since the reflected part of the incident wave is not contained in $t$. The derivation of Eq. (2) can, however, be easily adapted by replacing $S$ with $t$ and $\omega$ with $y_{b}$ in Eq. (1) (see also Ref. [44]). Most importantly, the resulting non-Hermitian GWS operator,

$$
q_{y_{b}}:=-i t^{-1} \frac{d t}{d y_{b}},
$$

inherits the property from its Hermitian counterpart $Q_{y_{b}}$ that its eigenstates are invariant with respect to a small change in the parameter $y_{b}$. In contrast to eigenstates of $Q_{y_{b}}$, the eigenstates of $q_{y_{b}}$ feature injection only from one lead, i.e., $\vec{u}_{n} \rightarrow \vec{u}_{n, L}$. The transmitted state, i.e., the outgoing state to the right, can be calculated via $\vec{v}_{R}=t \vec{u}_{L}$. Specifically, when adapting Eq. (2) to feature the complex eigenvalues $\vartheta_{n}^{y_{b}}=\beta_{n}^{y_{b}}+i \kappa_{n}^{y_{b}}$ of $q_{x_{b}}$, we have for the corresponding transmitted states

$$
\vec{v}_{n, R}\left(y_{b}+\Delta y_{b}\right) \approx e^{\left(i \beta_{n}^{y_{b}}-\kappa_{n}^{y_{b}}\right) \Delta y_{b}} \vec{v}_{n, R}\left(y_{b}\right) .
$$

Since the construction of the operator $q_{y_{b}}$ involves only the transmission matrix (the reflected part is omitted), its complex eigenvalues $\vartheta_{n}^{y_{b}}$ no longer correspond directly to the local momentum shift $\Delta k^{b}$ at the brass scatterer (see details in the Supplemental Material [38]). We do find, however, that a strong correlation between these two quantities persists (see Fig. S3 in Ref. [38]). Since this trace in the transmitted signal appears both in the phase and amplitude of a $q_{y_{b}}$ eigenstate as measured, respectively, by the real $\left(\beta_{n}^{y_{b}}\right)$ and imaginary $\left(\kappa_{n}^{y_{b}}\right)$ parts of $\vartheta_{n}^{y_{b}}$, we work with the absolute value of $\vartheta_{n}^{y_{b}}$ to quantify it. A more detailed analysis [38] shows that the correlation between $\left|\vartheta_{n}^{y_{b}}\right|$ and $\left|\Delta k^{b}\right|$ can be further increased by normalizing the latter 
term with the transmission (i.e., by working with $\left.\left|\Delta k^{b}\right| /\left|t^{2}\right|\right)$. We can thus apply the concept we derived for the Hermitian GWS operator $Q_{y_{b}}$ also to its nonHermitian counterpart $q_{y_{b}}$ with the essential difference being that the eigenvalues are now complex and sorted by their absolute value.

Following this protocol also in the experiment, we first measure the transmission amplitudes $t_{j i}$ for two slightly different transverse positions of the brass scatterer $\left( \pm \Delta y_{b}\right)$. We then determine the GWS operator $q_{y_{b}}$ by replacing the derivative in Eq. (4) with a finite-difference approximation based on the difference between the two prior transmission matrix measurements. In the next step, we evaluate the eigenstates of $q_{y_{b}}$ and inject them directly through the antenna array at the input port. To test if our focusing protocol works successfully, we then measure the intensity distribution of the microwave field in the vicinity of the brass scatterer by an additional scanning antenna. This antenna is attached to a movable arm and enters through a grid of holes (grid spacing $5 \mathrm{~mm} \times 5 \mathrm{~mm}$, hole diameter $2 \mathrm{~mm}$ ) in the top plate of the waveguide (extending $3 \mathrm{~mm}$ into the cavity). The obtained intensity distributions for the eigenvectors with the three largest and the three smallest eigenvalues (in absolute magnitude) are shown in Figs. 2 and 3, respectively. The displayed intensity profiles demonstrate very clearly that the largest eigenvalues correspond to states focusing on the target, see top row in Fig. 2. The eigenstates with the smallest eigenvalues, in turn, produce intensity patterns which are reduced almost to noise level in

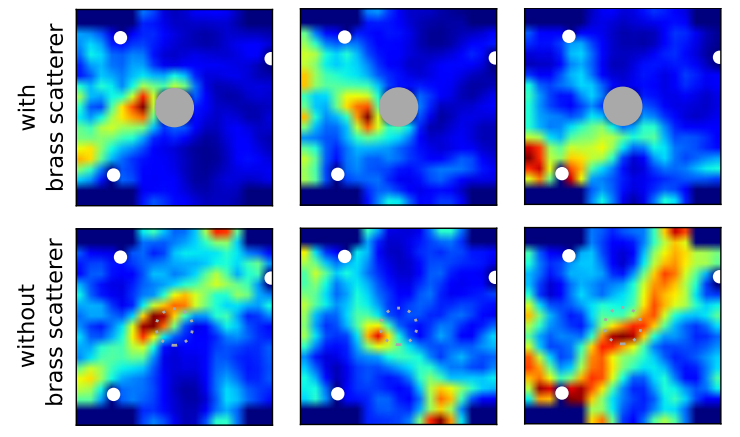

FIG. 2. Measured spatial distribution of the microwave intensity (see Supplemental Material [38]) inside the disordered scattering system realized in the experiment. The region shown is a zoom on the vicinity of the movable brass scatterer in the middle (as highlighted in the top panel of Fig. 1). In the top row this brass scatterer is included and in the bottom row it is removed. In both cases we display the eigenstates of $q_{y_{b}}$ with the largest eigenvalues $\left|\vartheta_{n}^{y_{b}}\right|=96.9,81.6$, and 66.9 [a.u.] from left to right. We can clearly observe that the intensity distribution is enhanced in the region around the brass scatterer (top row), such that removing the scatterer changes the intensity pattern strongly (bottom row). In each of the plots (also in Fig. 3) the color scale has been adjusted to match the maximum intensity (shown in dark red). the vicinity of the target, see top row in Fig. 3. As an additional test of our protocol, we also recorded the wave intensity patterns after removing the brass scatterer altogether. In the case of the focusing states such an intervention drastically changes the overall configuration of the intensity profiles, with intensity maxima near the position of the removed scatterer remaining clearly visible, see bottom row in Fig. 2. In the case of the states that avoid the brass scatterer, the wave intensity pattern remains almost unchanged as a whole when the scatterer is removed, see bottom row in Fig. 3. For the sake of clarity we emphasize here that the measurement of the intensity distribution in the interior of the investigated system serves only for demonstration purposes and is not necessary for implementing our protocol in the first place.

To summarize, we present here an extension of the Wigner-Smith time-delay operator to a whole class of operators with the exciting property of providing eigenstates that focus on or avoid a designated target inside a disordered medium. These generalized Wigner-Smith operators require the information stored in a system's scattering matrix as measured already in acoustics [45], seismology [46], and recently also in optics [1,2]. As a "guidestar" [47] for focusing deep in the multiple scattering regime, we use a movable scatterer inside the medium whose spatial shift leaves conspicuous traces in the measured transmission matrix that we exploit for our protocol. In the practical applications that we envision for future implementations, the spatial shift of the target scatterer could, e.g., result from the self-propelled movement of an object inside an otherwise static medium or be excited from the outside through an ultrasound focal spot that can be conveniently scanned through the medium (see Refs. [18,20] for recent implementations and Ref. [47] for a review). Our approach has the advantage that it works without phase conjugation and that the degree of focusing can be tuned up to the point where a target inside a medium can be entirely avoided rather than focused on. These features may be attractive for
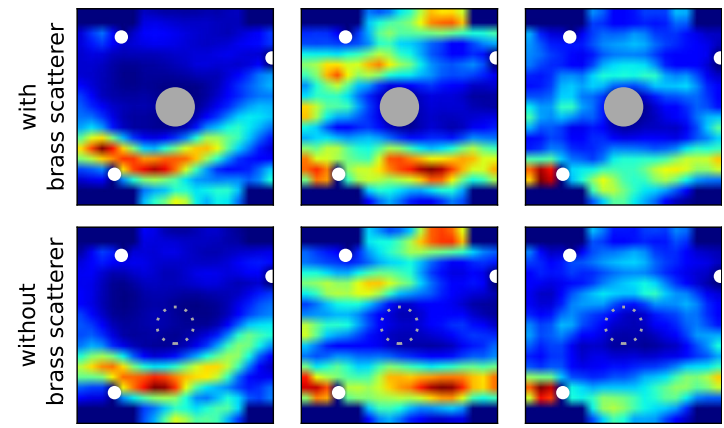

FIG. 3. Same as Fig. 2, but for the eigenstates of $q_{y_{b}}$ with the smallest eigenvalues $\left|\vartheta_{n}^{y_{b}}\right|=1.9,2.1$, and 6.0 [a.u.] from left to right. The measured intensity pattern clearly avoids the brass scatterer in the middle (top row), such that removing the scatterer leaves the intensity pattern almost unchanged (bottom row). 
novel imaging techniques in disordered media [48-50], including the case where certain parts of an imaged tissue should not get exposed to radiation. While the presented experiment using ten guided modes serves as a proofof-principle demonstration, we expect that the full potential of our method can be exploited once many modes are accessible as in the optical domain [3,4], see Figs. S4-S7 in Ref. [38].

P. A., A. B., M. K., and S.R. are supported by the Austrian Science Fund (FWF) through Projects No. SFB-NextLite F49-P10 and No. I 1142- N27 (GePartWave). J.B. and U. K. would like to thank the ANR for funding through Project GePartWave (ANR-12IS04-0004-01) and the European Commission through the H2020 programme by the Open Future Emerging Technology "NEMF21" Project (664828). The computational results presented in the Supplemental Material were achieved in part using the Vienna Scientific Cluster (VSC).

[1] S. M. Popoff, G. Lerosey, R. Carminati, M. Fink, A. C. Boccara, and S. Gigan, Measuring the Transmission Matrix in Optics: An approach to the Study and Control of Light Propagation in Disordered Media, Phys. Rev. Lett. 104, 100601 (2010).

[2] H. Yu, T. R. Hillman, W. Choi, J. O. Lee, M. S. Feld, R. R. Dasari, and Y. K. Park, Measuring Large Optical Transmission Matrices of Disordered Media, Phys. Rev. Lett. 111, 153902 (2013).

[3] A. P. Mosk, A. Lagendijk, G. Lerosey, and M. Fink, Controlling waves in space and time for imaging and focusing in complex media, Nat. Photonics 6, 283 (2012).

[4] S. Rotter and S. Gigan, Light fields in complex media: Mesoscopic scattering meets wave control, Rev. Mod. Phys. 89, 015005 (2017).

[5] I. M. Vellekoop, A. Lagendijk, and A. P. Mosk, Exploiting disorder for perfect focusing, Nat. Photonics 4, 320 (2010).

[6] I. M. Vellekoop and A. P. Mosk, Focusing coherent light through opaque strongly scattering media, Opt. Lett. 32, 2309 (2007).

[7] O. Katz, E. Small, Y. Bromberg, and Y. Silberberg, Focusing and compression of ultrashort pulses through scattering media, Nat. Photonics 5, 372 (2011).

[8] D. J. McCabe, A. Tajalli, D. R. Austin, P. Bondareff, I. A. Walmsley, S. Gigan, and B. Chatel, Spatio-temporal focusing of an ultrafast pulse through a multiply scattering medium, Nat. Commun. 2, 447 (2011).

[9] J. Aulbach, A. Bretagne, M. Fink, M. Tanter, and A. Tourin, Optimal spatiotemporal focusing through complex scattering media, Phys. Rev. E 85, 016605 (2012).

[10] S. Popoff, G. Lerosey, M. Fink, A. C. Boccara, and S. Gigan, Image transmission through an opaque material, Nat. Commun. 1, 81 (2010).

[11] M. Mounaix, H. Defienne, and S. Gigan, Deterministic light focusing in space and time through multiple scattering media with a time-resolved transmission matrix approach, Phys. Rev. A 94, 041802 (2016).
[12] M. Mounaix, D. Andreoli, H. Defienne, G. Volpe, O. Katz, S. Grésillon, and S. Gigan, Spatiotemporal coherent control of light through a multiple scattering medium with the multispectral transmission matrix, Phys. Rev. Lett. 116, 253901 (2016).

[13] H. P. Paudel, C. Stockbridge, J. Mertz, and T. Bifano, Focusing polychromatic light through strongly scattering media, Opt. Express 21, 17299 (2013).

[14] J. Aulbach, B. Gjonaj, P. M. Johnson, A. P. Mosk, and A. Lagendijk, Control of Light Transmission through Opaque Scattering Media in Space and Time, Phys. Rev. Lett. 106, 103901 (2011).

[15] I. M. Vellekoop, E. G. van Putten, A. Lagendijk, and A. P. Mosk, Demixing light paths inside disordered metamaterials, Opt. Express 16, 67 (2008).

[16] J. Aulbach, B. Gjonaj, P. Johnson, and A. Lagendijk, Spatiotemporal focusing in opaque scattering media by wave front shaping with nonlinear feedback, Opt. Express 20, 29237 (2012).

[17] T. Chaigne, O. Katz, A. Boccara, M. Fink, E. Bossy, and S. Gigan, Controlling light in scattering media non-invasively using the photoacoustic transmission matrix, Nat. Photonics 8, 58 (2014).

[18] X. Xu, H. Liu, and L. V. Wang, Time-reversed ultrasonically encoded optical focusing into scattering media, Nat. Photonics 5, 154 (2011).

[19] C. Ma, X. Xu, Y. Liu, and L. V. Wang, Time-reversed adapted-perturbation (TRAP) optical focusing onto dynamic objects inside scattering media, Nat. Photonics 8, 931 (2014).

[20] B. Judkewitz, Y. M. Wang, R. Horstmeyer, A. Mathy, and C. Yang, Speckle-scale focusing in the diffusive regime with time reversal of variance-encoded light (TROVE), Nat. Photonics 7, 300 (2013).

[21] E. H. Zhou, H. Ruan, C. Yang, and B. Judkewitz, Focusing on moving targets through scattering samples, Optica 1, 227 (2014).

[22] E. P. Wigner, Lower limit for the energy derivative of the scattering phase shift, Phys. Rev. 98, 145 (1955).

[23] F. T. Smith, Lifetime matrix in collision theory, Phys. Rev. 118, 349 (1960).

[24] T. Kottos, Statistics of resonances and delay times in random media: beyond random matrix theory, J. Phys. A 38, 10761 (2005).

[25] R. Pazourek, S. Nagele, and J. Burgdörfer, Attosecond chronoscopy of photoemission, Rev. Mod. Phys. 87, 765 (2015).

[26] S. Fan and J. M. Kahn, Principal modes in multimode waveguides, Opt. Lett. 30, 135 (2005).

[27] S. Rotter, P. Ambichl, and F. Libisch, Generating Particlelike Scattering states in Wave Transport, Phys. Rev. Lett. 106, 120602 (2011).

[28] J. Carpenter, B. J. Eggleton, and J. Schröder, Observation of Eisenbud-Wigner-Smith states as principal modes in multimode fibre, Nat. Photonics 9, 751 (2015).

[29] W. Xiong, P. Ambichl, Y. Bromberg, B. Redding, S. Rotter, and H. Cao, Spatiotemporal Control of Light Transmission through a Multimode Fiber with Strong Mode Coupling, Phys. Rev. Lett. 117, 053901 (2016). 
[30] B. Gérardin, J. Laurent, P. Ambichl, C. Prada, S. Rotter, and A. Aubry, Particlelike wave packets in complex scattering systems, Phys. Rev. B 94, 014209 (2016).

[31] Y. V. Fyodorov and H.-J. Sommers, Statistics of resonance poles, phase shifts and time delays in quantum chaotic scattering: Random matrix approach for systems with broken time-reversal invariance, J. Math. Phys. (N.Y.) 38, 1918 (1997).

[32] P. W. Brouwer, K. M. Frahm, and C. W. J. Beenakker, Quantum Mechanical Time-Delay Matrix in Chaotic Scattering, Phys. Rev. Lett. 78, 4737 (1997).

[33] D. V. Savin and H.-J. Sommers, Delay times and reflection in chaotic cavities with absorption, Phys. Rev. E 68, 036211 (2003).

[34] J. Böhm, A. Brandstötter, P. Ambichl, S. Rotter, and U. Kuhl, Particlelike scattering states in a microwave cavity, arXiv:1706.08926.

[35] P. A. Lee and A. D. Stone, Universal Conductance Fluctuations in Metals, Phys. Rev. Lett. 55, 1622 (1985).

[36] T. Gorin, T. Prosen, T. H. Seligman, and M. Žnidarič, Dynamics of Loschmidt echoes and fidelity decay, Phys. Rep. 435, 33 (2006).

[37] Y. V. Fyodorov and D. V. Savin, Statistics of Resonance Width Shifts as a Signature of Eigenfunction Nonorthogonality, Phys. Rev. Lett. 108, 184101 (2012).

[38] See Supplemental Material at http://link.aps.org/ supplemental/10.1103/PhysRevLett.119.033903 which includes Refs. [26, 39-41] for a detailed discussion of the GWS operator and its eigenvalues as well as for additional numerical simulations.

[39] S. Rotter, J.-Z. Tang, L. Wirtz, J. Trost, and J. Burgdörfer, Modular recursive Green's function method for ballistic quantum transport, Phys. Rev. B 62, 1950 (2000).
[40] F. Libisch, S. Rotter, and J. Burgdörfer, Coherent transport through graphene nanoribbons in the presence of edge disorder, New J. Phys. 14, 123006 (2012).

[41] P. Ambichl, Ph.D. thesis, Vienna University of Technology, Institute for Theoretical Physics, 2016.

[42] J. Böhm and U. Kuhl, Wave front shaping in quasi-onedimensional waveguides, in 2016 IEEE Metrology for Aerospace (MetroAeroSpace) (IEEE, New York, 2016), pp. 182-186.

[43] A. Peña, A. Girschik, F. Libisch, S. Rotter, and A. A. Chabanov, The single-channel regime of transport through random media, Nat. Commun. 5, 3488 (2014).

[44] A. A. Juarez, C. A. Bunge, S. Warm, and K. Petermann, Perspectives of principal mode transmission in modedivision-multiplex operation, Opt. Express 20, 13810 (2012).

[45] B. Gérardin, J. Laurent, A. Derode, C. Prada, and A. Aubry, Full Transmission and Reflection of Waves Propagating through a Maze of Disorder, Phys. Rev. Lett. 113, 173901 (2014).

[46] E. Larose, L. Margerin, B. A. van Tiggelen, and M. Campillo, Weak Localization of Seismic Waves, Phys. Rev. Lett. 93, 048501 (2004).

[47] R. Horstmeyer, H. Ruan, and C. Yang, Guidestar-assisted wavefront-shaping methods for focusing light into biological tissue, Nat. Photonics 9, 563 (2015).

[48] I. M. Vellekoop and C. M. Aegerter, Scattered light fluorescence microscopy: Imaging through turbid layers, Opt. Lett. 35, 1245 (2010).

[49] J. Bertolotti, E. G. van Putten, C. Blum, A. Lagendijk, W. L. Vos, and A. P. Mosk, Non-invasive imaging through opaque scattering layers, Nature (London) 491, 232 (2012).

[50] J. A. Newman, Q. Luo, and K. J. Webb, Imaging Hidden Objects with Spatial Speckle Intensity Correlations over Object Position, Phys. Rev. Lett. 116, 073902 (2016). 